\title{
A double blind trial of sulpiride in Huntington's disease and tardive dyskinesia
}

\author{
NIALL QUINN, C DAVID MARSDEN \\ From the University Department of Neurology, Institute of Psychiatry and King's College Hospital Medical \\ School, London, UK
}

SUMMARY Eleven patients with Huntington's disease and nine patients with tardive dyskinesia participated in a randomised double-blind crossover trial of sulpiride (as sole antidopaminergic therapy) versus placebo. Although functional improvement was not seen in Huntington's disease patients, sulpiride reduced movement count and total dyskinesia score in both conditions. Sulpiride differs pharmacologically in several respects from conventional neuroleptics, and has not been convincingly shown to cause tardive dyskinesia. Among currently available treatments, it may therefore be considered a drug of choice for treatment of tardive dyskinesia.

Over-activity (relative or absolute) of cerebral dopaminergic mechanisms in the basal ganglia is believed to play a role in the genesis of abnormal movements in both Huntington's disease and tardive dyskinesia. ${ }^{12}$ The abnormal movements in both conditions are frequently reduced by conventional neuroplastic drugs of the phenothiazine, butyrophenone and thioxanthene classes, but often at the expense of drug-induced Parkinsorism. In addition, the long-term treatment of patients with tardive dyskinesia and Huntington's disease with these drugs ultimately may lead to a super-imposed tardive movement disorder.

Since the identification and classification of D-1 and D-2 dopamine receptors, ${ }^{3}$ there has been renewed interest in attempts to separate desirable and undesirable effects of both dopamine agonist and antagonist drugs. ${ }^{4}$ Most neuroleptics have actions at both D-1 and D- 2 receptor sites. Drugs of the substituted benzamide group are potent and selective antogonists at $D-2$ receptor sites. They do not inhibit dopamine-stimulated adenylate cyclase activity in vitro except at exceedingly high concentrations. ${ }^{5}$ This selectivity of action may be of advantage in an anti-dyskinetic drug.

One of the benzamides, sulpiride, has been widely used in Europe as an anti-psychotic and antidepressant drug. We have found no published report

Address for reprint requests: Professor CD Marsden, University Department of Neurology, Institute of Psychiatry, De Crespigny Park, London E5, UK

Received 18 November 1983. Accepted 8 December 1983 of a formal trial of sulpiride in Huntington's disease and only two ${ }^{67}$ in tardive dyskinesia. We have therefore evaluated the use of sulpiride as sole antidyskinetic treatment in patients with Huntington's disease and tardive dyskinesia.

\section{Patients and methods}

\section{Patients}

Eleven patients with Huntington's disease, seven male and four female, participated in the study. They were aged from 37-67 years (mean 53), and had displayed signs of the disorder for 3-16 years (mean 6.7). The stage of the disease on the Shoulson \& Fahn scale ${ }^{8}$ was from 3-5 (mean 3.8). Eight were long-stay in-patients and three were cared for at home.

Of the nine patients with tardive dyskinesia, two were male and seven female. Their ages ranged from 52-82 years (mean 61). Six were long-stay in-patients, three were out-patients. Five had received neuroleptic drugs for chronic schizophrenia, one for schizo-effective disorder, one for bipolar affective disorder, one for agitated depression, and one for a state of chronic agitation.

\section{Protocol}

The study protocol, which was of randomised double-blind cross-over design, was approved by the Hospitals' Ethical Committees. All patients underwent a two-week run-in period, free of anti-dopaminergic medication (any other treatment being continued unchanged throughout). This necessitated stopping existing neuroleptic treatment in seven of the eleven Huntington's disease patients and two of the nine tardive dyskinesia patients. At the end of this period, active sulpiride or matching placebo were each administered for a four-week period. Treatment periods were separated by a one-week "wash-out" interval. During the first week of each treatment phase, patients 
received either $300 \mathrm{mg}$ sulpiride per day (100 mg three times daily) or matching placebo. For weeks 2,3 and 4 the doses were 600,900 and $1200 \mathrm{mg} /$ day (divided into three doses) respectively. In the event of troublesome side effects, there was provision for the dose of drug to be maintained rather than increased, or even reduced. If patients showed marked clinical deterioration during the "wash-out" period or either of the treatment periods, they passed prematurely into the next phase of the trial. Thus, by the end of each month the patients were receiving close to their optimum dose within the range $200-1200 \mathrm{mg}$ per day of sulpiride (or placebo equivalent). The randomisation code was broken only after all patients had completed the study.

\section{Assessment}

Patients were rated at the end of weeks 2 and 4 of each four-week treatment period. Three different rating criteria were used.

(A) Movement count. During the run-in period, a single easily identifiable abnormal movement, for example lip pouting occurring with a measurable frequency (neither too low nor too high) was selected. During the treatment phases, the mean number of such movements occurring per minute over two separate sixty second intervals, with the patient at rest, was recorded.

(B) Chorea severity score. A combined score ${ }^{9}$ for severity of abnormal movements (frequency considered together with amplitude) for eight body parts, together with assessments of speech, gait, postural stability and manual dexterity was allocated by the examiner (NPQ) at the end of a 20 minute period covering the whole of the rating. Maximum possible score on this scale was 59.

(C) Functional score. A functional disability scale for Huntington's disease patients was devised which recorded information about the patient's capacities for walking, dressing, swallowing, feeding, speech and prominence of chorea. Patients were scored in consultation with spouse, or attendant nursing staff, on the basis of their performance over the preceding week. Maximum possible disability score was 30 .

The Wilcoxon signed rank test for non-parametric data was used for statistical comparisons.

\section{Results}

Individual patient scores at the end of the fourth
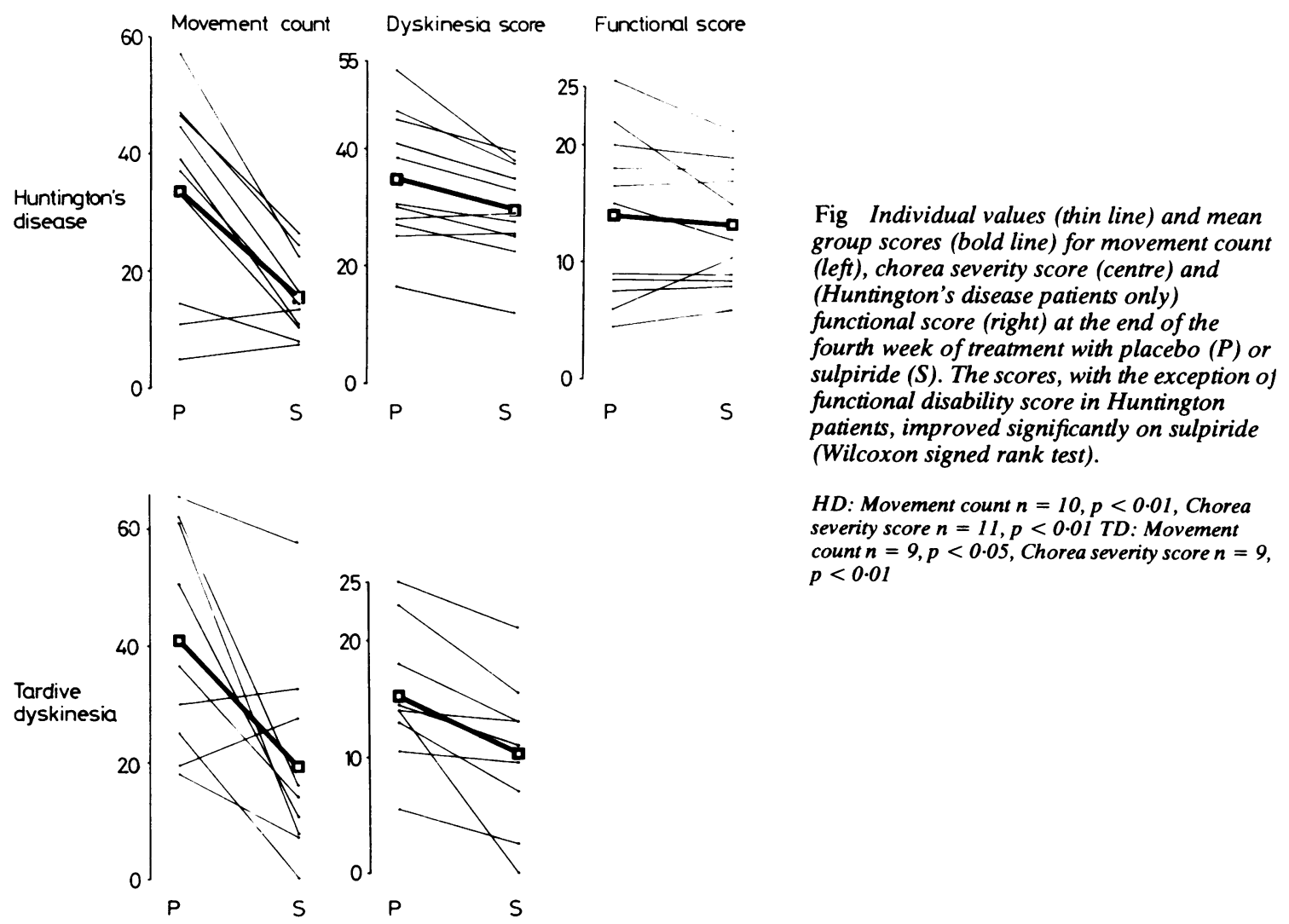

HD: Movement count $n=10, p<0.01$, Chorea severity score $n=11, p<0.01$ TD: Movement count $n=9, p<0.05$, Chorea severity score $n=9$, $p<0.01$ 
Table Side effects attributable to sulpiride in patients with Huntington's disease $(H D)(n=11)$ or tardive dyskinesia (TD) $(n=9)$

\begin{tabular}{llll}
\hline Side effect & Condition & $\begin{array}{l}\text { No. of patients } \\
\text { with side effect }\end{array}$ & $\begin{array}{l}\text { Mean dose (mg/day) } \\
\text { at which side effect } \\
\text { furst noted }\end{array}$ \\
\hline Drowsiness & HC & 5 & 800 \\
Increasing Parkinsonism & TD & 2 & 600 \\
Increased akathisia & TD & 1 & 7 \\
Decreased akathisia & TD & 1 & 600 \\
Amenorrhoea & TD & 1 & 600 \\
\hline
\end{tabular}

week of treatment with sulpiride and placebo are presented in the figure.

\section{HUNTINGTON'S DISEASE}

Median movement count for ten subjects (the eleventh was too drowsy at rest on active sulpiride) fell from 38 on placebo to 14 on sulpiride $(p<$ 0.01 ). Two mildly affected patients had more movements on active sulpiride than on placebo. Median chorea severity score fell from 33 to 29 ( $p<$ 0.01). Two patients had higher scores on sulpiride than on placebo. Median functional score did not change significantly between placebo (15) and sulpiride (12) $(p>0.05)$.

Eight patients reached $1200 \mathrm{mg}$ of sulpiride per day, two $900 \mathrm{mg}$ per day, and one could tolerate only $200 \mathrm{mg}$ per day. Nine patients reached the week 4 dosage placebo, but two only reached week 2 dosage because of worsening disability.

\section{TARDIVE DYSKINESIA}

Median movement count decreased from 36.5 on placebo to 14 on sulpiride $(p<0.05)$. In one patient marked abnormal movements were completely suppressed, but in two the movement count increased slightly on active sulpiride. Dyskinesia severity score decreased in all individual patients, and also in the group as a whole (median score 14 on placebo, 11 on sulpiride, $p<0.01$ ). Five patients reached $1200 \mathrm{mg}$ of sulpiride per day, three could tolerate only $600 \mathrm{mg}$, and one only $200 \mathrm{mg}$ per day. All but two patients, whose movements were markedly worse after week 2 , reached week 4 placebo dosage.

\section{SIDE EFFECTS}

The table shows the incidence of side effects attributable to sulpiride, together with the mean dose of the drug at which the side effect was first noted. Patients with Huntington's disease generally tolerated sulpiride better, and attained higher mean daily dosages $(1055 \mathrm{mg} /$ day $)$ than those with tardive dyskinesia ( $890 \mathrm{mg} /$ day). Drowsiness was their principal complaint. The low incidence of identifiable drug-induced Parkinsonism in Huntington's disease patients may simply be a reflection of the difficulty of recognising it in the presence of marked chorea. By contrast, signs of emerging or worsening Parkinsonism were seen in seven of the nine tardive dyskinesia patients. In five of them it was evident after only two weeks treatment with sulpiride, and in three (all with mild pre-existing Parkinsonism) it constituted an active symptomatic complaint preventing further increase in dosage. In general, those tardive patients whose dyskinesia was most improved were also those in whom Parkinsonism appeared or worsened.

\section{Discussion}

In this study sulpiride given as sole antidopaminergic treatment reduced abnormal movements in patients with Huntington's disease and tardive dyskinesia. In most patients with Huntington's disease, however, this change was not accompanied by functional improvement. Mildly affected patients tended to be worse on one or more of the rating scales when taking sulpiride as opposed to placebo, an occurrence also witnessed by Roos et al ${ }^{10}$ during a trial of tiapride. In several patients, the three different rating parameters did not necessarily all improve or worsen together, and they sometimes changed in opposite directions; hence the importance of scoring more than one clinical parameter in such studies.

Sulpiride is known to be an effective antipsychotic," and this study has confirmed that in most patients it will also reduce and sometimes completely suppress the abnormal movements of tardive dyskinesia. The clinical use of sulpiride alone as an anti-psychotic and, in low dosage, as an antidepressant, has not generated conclusive evidence of the induction of tardive dyskinesia by the drug. This feature, together with the pharmacological differences from other neuroleptics mentioned above, makes it an attractive choice for treatment of this condition in certain selected cases. However, another substituted benzamide, metoclopramide, is recognised as a cause of both tardive dyskinesia and drug-induced Parkinsonism. ${ }^{1213}$ In the case of sul- 
piride, there is no doubt that the drug induces Parkinsonism, as was evident in the present study. Whether it can cause tardive dyskinesia is uncertain. The issue is clouded in psychotic patients by the prior or concurrent administration of other neuroleptics, whilst the absence of tardive dyskinesia in patients treated for depression could be simply a reflection of the low dosages used combined with low penetration of the drug into the brain.

Tardive dyskinesia usually does not cause functional disability in daily living activities. Its impact on patients can be, nonetheless, disastrous, either in terms of personal distress or in compromising their social rehabilitation. In addition, a number of these patients need continued anti-psychotic medication because of the risk of a recurrence of their psychiatric symptoms. Within the currently available range of anti-psychotic agents, sulpiride may be the drug of choice for the treatment of tardive dyskinesia in patients needing continued anti-psychotic treatment. Caution should be exercised, however, particularly in using the drug to treat tardive dyskinesia in patients who do not need continued antipsychotic medication. In these subjects, sulpiride should be withdrawn, or the dosage reduced, at intervals of six to twelve months in order to establish whether the underlying movement disorder is still present, improved, or worse.

We gratefully acknowledge the gift of sulpiride from Delagrange Laboratories, Paris, and the help and cooperation of patients and staff at Fairfield Hospital, Hertfordshire, and the Sue Ryder Home, Stagenhoe Park, Hertfordshire.
References

' Spokes EGS. Dopamine in Huntington's disease: A study of postmortem brain tissue. Adv Neurol 1979;23:481-93.

${ }^{2}$ Clow A, Theodorou A, Jenner P, Marsden CD. Cerebral dopamine function in rats following withdrawal from one year of continuous neuroleptic administration. Eur J Pharm 1980;63:145-57.

${ }^{3}$ Kebabian JW, Calne DB. Multiple receptors for dopamine. Nature 1979;277:93-6.

${ }^{4}$ Schachter M, Bedard P, Debono AG, et al. The role of D-1 and D-2 receptors. Nature 1980;286:157-9.

5 Jenner P, Marsden CD. The mechanism of action of substituted benzamine drugs. In: Spano PF, Trabucchi M, Corsini GU, et al, eds. Sulpiride and other Benzamides. pp. 119-147, Milan: Italian Brain Research Foundation Press, 1979.

- Casey DE, Gerlach J, Simmelsgaard H. Sulpiride in tardive dyskinesia. Psychopharmacology 1979;66:73-7.

${ }^{7}$ Haggstrom J-E. Sulpiride in tardive dyskinesia. Curr Ther Res 1980;27:164-9.

${ }^{8}$ Shoulson I, Fahn S. Huntington's disease: clinical care and evaluation. Neurology (Minneap) 1979;29:1-3.

${ }^{9}$ Marsden CD, Schachter M. Assessment of extrapyramidal disorders. Br J Clin Pharmacol 1981;11:129-51.

${ }^{10}$ Roos RAC, Buruma OJS, Bruyn GW, Kemp B, Van Der Velde EA. Tiapride in the treatment of Huntington's chorea. Acta Neurol Scand 1982;65:45-50.

" Edwards JG, Alexander JR, Alexander MS, Gordon A, Zutchi $T$. Controlled trial of sulpiride in chronic schizophrenic patients. $\mathrm{Br} J$ Psychiatry 1980; 137:522-9.

${ }^{12}$ Lavy S, Melamed E, Penchas S. Tardive dyskinesia associated with metoclopramide. $\mathrm{Br}$ Med J 1978;i:77-78.

${ }^{13}$ Indo T, Ando K. Metoclopramide-induced Parkinsonism. Clinical characteristics of ten cases. Arch Neurol 1982;39:494-6. 